\title{
Enhanced lumped circuit model for smart nanocomposite cement-based sensors under dynamic compressive loading conditions
}

\author{
Enrique García-Macías ${ }^{\mathrm{a}, *}$, Austin Downey $^{\mathrm{b}}$, Antonella D’Alessandroc ${ }^{\mathrm{c}}$, Rafael Castro-Triguero ${ }^{\mathrm{d}}$, \\ Simon Laflamme ${ }^{\mathrm{b}}$, Filippo Ubertini ${ }^{\mathrm{c}}$ \\ ${ }^{a}$ Department of Continuum Mechanics and Structural Analysis, School of Engineering, University of Seville, Camino de los Descubrimientos \\ s/n, E-41092-Seville, Spain \\ ${ }^{b}$ Department of Civil, Construction, and Environmental Engineering, Iowa State University, 394 Town Engineering, Ames, IA 50011 \\ ${ }^{c}$ Department of Civil and Environmental Engineering, University of Perugia, Via G Duranti 93, Perugia 06125, Italy \\ ${ }^{d}$ Department of Mechanics, University of Cordoba, Campus de Rabanales, Cordoba, CP 14071, Spain
}

\begin{abstract}
Smart composite nanostructured materials represent one of the fastest-growing areas of interest among scientists in recent years and, in particular, Carbon NanoTube (CNT) cement-based composites are attracting more and more attention. These composites exhibit self-sensing capabilities providing measurable variations of their electrical properties under the application of mechanical deformations. Together with this exceptional property, the similarity and compatibility between these composites and structural concrete suggest the possibility of developing distributed embedded strain-sensing systems with substantial improvements in the cost-effectiveness in applications to large-scale concrete structures. In order to design and optimize CNT reinforced cement based dynamic sensors, it is fundamental to develop theoretical models capable of simulating the relationship between dynamic mechanical strains and the effective electrical conductivity. This paper presents an electromechanical modeling of the Direct Current (DC) electrical resistance of CNT reinforced cement paste sensors based on a piezoelectric/piezoresistive lumped circuit. The model represents an enhanced version and a generalization of another model previously proposed by the authors. Previously published experimental results have been used as validation benchmark. In particular, experimental tests concerning the characterization of the step response under unloaded conditions, steady state response under harmonic loadings and sweep analyses are considered. The results demonstrate that the newly proposed model is superior in comparison to the previous one in reproducing the dynamic response of the sensors when subjected to harmonic mechanical loads. Overall, an excellent agreement between theoretical predictions and experimental results is achieved.
\end{abstract}

Keywords:

Carbon nanotube, Cement-matrix composites, Lumped equivalent circuit, DC electrical resistance, Smart concrete, Structural Health Monitoring

\section{Introduction}

The critical socio-economical and environmental importance of civil infrastructures makes condition-based maintenance and Structural Health Monitoring (SHM) of the utmost relevance for structural engineering. A large portion of the worldwide civil infrastructures is constituted by concrete. However, there is a growing concern

\footnotetext{
${ }^{*}$ Corresponding author.

Email address: egarcia28@us . es (Enrique García-Macías)
} 
with the lifetime of these structures because of concrete's wide material discreteness and complex service environments $[1,2]$. Currently, the majority of the methods used for the local monitoring of concrete structures (e.g. strain gauges, optic sensors, piezoelectric ceramic, shape memory alloy, etc.) do not offer efficient solutions for embedded monitoring systems capable of getting information about the structural integrity and detecting damage [3]. The new development of multifunctional and smart materials, and particularly electrically conductive CNT cement-based composites, opens up a wide range of possibilities in the realm of SHM [4-6]. These composites exhibit strain-sensing capabilities (i.e. composites themselves also behave as sensors) by means of measurable variations of their electrical properties under applied mechanical deformations [7-9]. This exceptional property, together with the similarity and compatibility between these composites and structural concrete, can be used to develop distributed embedded strain-sensing systems with substantial improvements in the cost-effectiveness in applications to large-scale concrete structures [10-16]. In this regard, some of the authors recently developed a Carbon Nanotube Cement-based Sensor (CNTCS) [5, 17, 18]. The sensor, consisting of a cement paste doped with Multi-Walled Carbon Nanotubes (MWCNTs), is a transducer of external mechanical strains into a measurable variation of the electrical resistance. Previous studies on CNTCSs [5, 18] have already demonstrated the potential of CNTCSs as dynamic strain sensors in concrete structures. However, in order to properly design and optimize the sensors, a tractable electromechanical model is yet to be fully developed.

Impelled by the promising potential of these composites as smart materials, most studies have focused on the experimental characterization and the development of new applications as sensors [4, 5, 18-24]. However, theoretical studies that help to understand the strain sensing capability of CNTCSs are still lacking. A percolation-like behavior has been widely used to explain the electrical conductivity of cement-based composites [25-27]. From a physical point of view, two different conductive mechanisms, namely electron hopping and conductive networking, contribute to the overall electric conductivity $[25,28-30]$. The percolation threshold indicates the critical volume fraction of CNTs at which adjacent fibers touch one another resulting in a continuous conductive path and a consequent drastic increase of the overall conductivity. Below percolation, only the transfer of electrons intra-tube or tube-tube is possible due to the large distances among CNTs. Once percolation has been reached, both mechanisms act simultaneously although conductive networks become dominant. Therefore, the origin of the self-sensing property is ascribed to the alteration of these mechanisms induced by mechanical strains. In particular, three major changes during stretching/compressing have been suggested in the literature [31-33]: (i) volume expansion, (ii) reorientation of fillers and (iii) changes in the percolation threshold. In this regard, it is worth noting the works of Deng and Zheng [34] and Takeda et al. [35] who presented a simplified micromechanics model to evaluate the effective electrical conductivity of CNT/polymer composites. This approach allowed us to distinguish the contribution of tunneling-type contacts and conductive networking, simulate percolation, conductivity anisotropy and waviness of CNTs with fairly good agreements with experimental data. Another influential contribution was made by Seidel and Lagoudas [36] and later extended by Feng and Jiang [37] who proposed an Eshelby-Mori-Tanaka micromechanics model for the study of the individual influence of the conductive mechanisms on the overall electrical conductivity of CNT/polymer composites. A similar framework was used by García et al. [33] for the modeling of CNT reinforced cement-based materials, namely cement paste, mortar and concrete, with the consideration of waviness and agglomeration effects. Work related to the strain effects on the conductivity is more limited. A Monte Carlo method was proposed by Lin et al. [38] to investigate the stretching/compression effects on the electrical properties of fiber-filled composites. Theodosiou and Saravanos [39] analyzed the piezoresistive response of CNTs with an atomistic model and the overall behavior of CNT-polymer composites at macroscale by a numerical percolation model. That research concluded that the nanotube resistance and the tunneling effect were the dominant mechanisms of strain-sensitivity of the composites. Tallman and Wang [40] extended the analytical framework developed by Takeda et al. [35] for the piezoresistivity modeling of CNT composites subjected to arbitrary three-dimensional strains. The Eshelby-Mori-Tanaka micromechanics model was also used by Feng and Jiang [31,41] and García et al. [42] to simulate the piezoresistivity of CNT reinforced polymers and cement-based materials, respectively. In the latter work, analytical and experimental results demonstrated that the largest strain-sensitivity is achieved at the percolation threshold. Another important issue investigated, also addressed by some other authors [31, 40, 43, 44], refers to the nonlinear relationship between ohmic resistance and externally applied strains. The results concluded that strain induced variations of the percolation threshold lead to a certain level of nonlinearity in the response, especially important for filler contents close to the percolation threshold.

Although a vast effort has been put into understanding the physical mechanisms governing the strain sensitivity of CNT reinforced nanocomposites, none of the above mentioned methodologies offers suitable approaches to analyzing the dynamic response, essential for applications in the form of dynamic strain sensors. Cement-based materials primarily behave as dielectrics. When a dielectric material is exposed to an electric field, the molecular dipoles tend to get oriented in the direction of the field so as to create an induced electric field opposite to the applied one. As a consequence of this phenomenon, termed polarization, the electric current decreases over time 
under the application of a constant potential difference $[45,46]$. Polarization, together with strain-varying electrical properties, must be taken into account when simulating DC resistivity measurements. To do so, equivalent lumped-circuit models are being imposed as they provide a feasible and tractable framework to simulate electric time-varying systems. The work of Kang et al. [47] who performed electrochemical impedance spectroscopy (EIS) testing to characterize the electrical properties of SWNT/PMMA sensor materials. On this basis, the authors proposed a modified Randle's circuit to represent the dynamic behavior of the sensors. Similarly to other works, strain sensing was enabled by a linear relationship between the variation of the relative change of the internal electrical resistance and the axial strain. This type of approaches neglects any kind of nonlinearity in the response. This limits the application of these models to small deformation ranges and filler contents away from the percolation threshold. A similar study is the one of Loh et al. [48] on SWNT-PSS/PVA thin films. An RC-circuit model was formulated from frequency-domain electrical impedance spectroscopy to fit the numerical results to the experimental data. The contact resistance, the double layer capacitance and the bulk resistance were defined exponentially decaying with time and a good agreement with the experiments was obtained. Also, Materazzzi et al. [49] conducted an experimental campaign to assess the change in the electrical resistance of CNTCSs under the action of sinusoidal compression loads. The analyses showed that the amplitude of the electrical resistance variation increases with the frequency of excitation. Following the trace of that work, D'Alessandro et al. [50] proposed a Randle's equivalent circuit based electromechanical modeling of CNTCSs, similar to the one proposed by Han et al. [51]. The lumped-circuit consisted of two resistors and a capacitor, accounting for the contact resistance (cables and electrodes), electric polarization and internal dissipation. An important conclusion was that the dynamic response of CNTCSs is not monochromatic, but rather contains superharmonics. However, while the presence of superharmonics was anticipated by the model, the increasing amplitude of the response with increasing frequency was not reproduced. Sanli et al. [52] proposed an RC equivalent circuit based on the impedance response of sensitive CNT/epoxy films. An interesting aspect of that work is the consideration of not only internal resistance dependence on external strain, but also strain-dependent capacitance.

In this paper, we present an electromechanical model for CNTCSs inspired by a universal equivalent circuit proposed by Wanson et al. [53]. The model represents an enhanced version and a generalization of the model previously proposed by some of the authors in [50]. The circuit consists of a resistor in series with two RC elements, standing for the contact resistance, an inter-particle matrix path, and the interface response, respectively. Based on experimental evidence, this paper proposes the first piezoelectric/piezoresistive approach in the literature for the dynamic modeling of CNTCSs. The piezoresistive effect is simulated by a linear variation of the bulk resistance with strain, and the piezoelectric effect is modeled as an additional strain induced current source. Previously published experimental results in [49] and [50] have been used as validation benchmark. In particular, experimental tests have been conducted to characterize the step response under unloaded conditions, steady state response under harmonic loadings and sweep analyses. The parameters have been fitted by least-square differences optimization and excellent agreements have been found with experimental data. The results demonstrated that the proposed model can obtain the superharmonic response of CNTCs under cyclic loading, as well as the amplification of the electrical signals with the frequency of excitation.

The paper is organized as follows. Section 2 reviews the formulation of the previous equivalent lumped-circuit and presents the proposed enhanced version. Section 3 describes the manufacturing process and the testing details. Section 4 provides details of the numerical results containing step response of unloaded specimens, harmonic excitation and sweep tests. Finally, Section 5 presents the main contributions of the paper.

\section{Equivalent electric circuit}

Prismatic samples of nanocomposite cement paste doped with CNTs are considered in this work and denoted as CNTCS. The samples exhibit a change in their electrical resistivity under applied mechanical deformation, what can be measured by connecting an external DAQ system to electrodes embedded in the specimens. To elucidate the electrical behavior of the CNTCS under applied mechanical deformations, the samples are connected to a power source providing a stabilized potential difference across their electrodes, $V$, and to a digital multimeter measuring the time trace of the current passing through the specimen, $I(t)$, as illustrated in Fig. 1, where $t$ is the time variable. This configuration is commonly referred to as two probe configuration. Coaxial cables are used to make connections so as to limit electromagnetic interference and noise. 

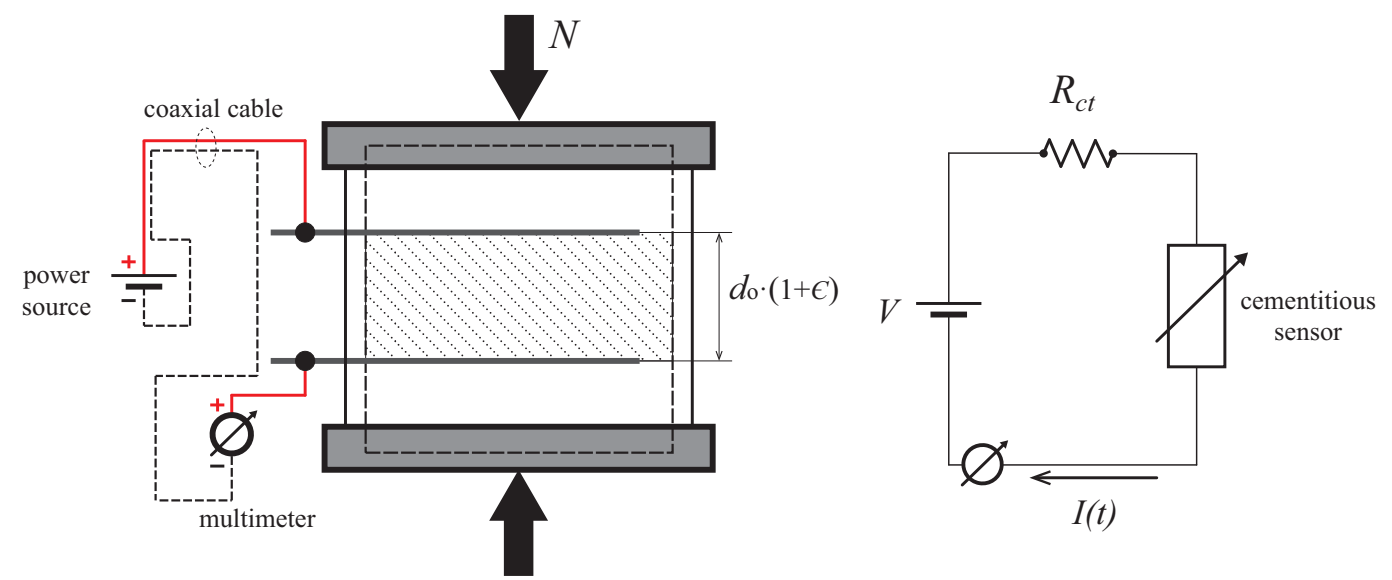

Figure 1: Layout of the two-probe method set-up.

As discussed above, many experimental studies have reported on the dynamic response of these smart materials, and they agree on two different phenomena:

- The response in terms of apparent electric resistance of CNTCS, that is, the ratio between applied voltage and measured current, subjected to a cyclic mechanical loading, $N$, is defined by a harmonic distortion with respect to the monochromatic input.

- The frequency response function (FRF) of the electric resistance of CNTCS, that is, the amplitude of the response over the amplitude of the mechanical input, increases with the frequency of excitation, i.e., the amplitude of the resistance time series increases with the frequency of excitation.

An adequate electromechanical modeling must simultaneously reproduce both phenomena.

\subsection{Piezoresistive equivalent circuit}

All the studies conducted so far have ascribed the strain-sensitivity of CNT reinforced nanocomposites to a piezoresistive behavior. The change in the electrical resistivity under applied mechanical strain is attributed to three main mechanisms: (i) strain-induced changes in the volume fraction, (ii) filler reorientation and, (iii) changes in the tunneling resistance [42]. All the previously proposed lumped-circuit models for the electromechanical characterization of CNTCSs are based on this principle and, thus, the strain-sensing capability is assumed to be only caused by changes in the internal resistance or capacitance $[47,48,50,51]$. Although some variants can be found in the literature, the main sensing cell is the one represented in Fig. 2(a). From this modeling approach, the active volume of the specimen, defined as the portion $d_{o}$ of the sensor in between the selected pair of electrodes, is modeled as a capacitor and a resistor in parallel. $R_{i p}$ and $C_{i p}$ denote internal resistance and capacitance, respectively, while $R_{c t}$ comprises contact resistances for cables, electrodes, and the specimens. In the case of the DC two-probe measurement method, represented in Fig. 1, the time evolution of the current through the sample is governed by the following differential equation [50]:

$$
\frac{\mathrm{d} I(t)}{\mathrm{d} t}+\frac{I(t)}{\tau}=\frac{V}{\tau \bar{R}}
$$

where $\bar{R}=R_{c t}+R_{i p}$, and

$$
\tau=\frac{C_{i p} R_{c t} R_{i p}}{\bar{R}}
$$

is the characteristic time of the circuit. 
(a)

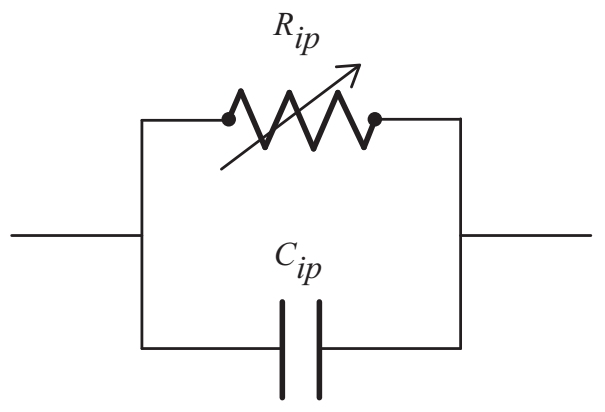

(b)
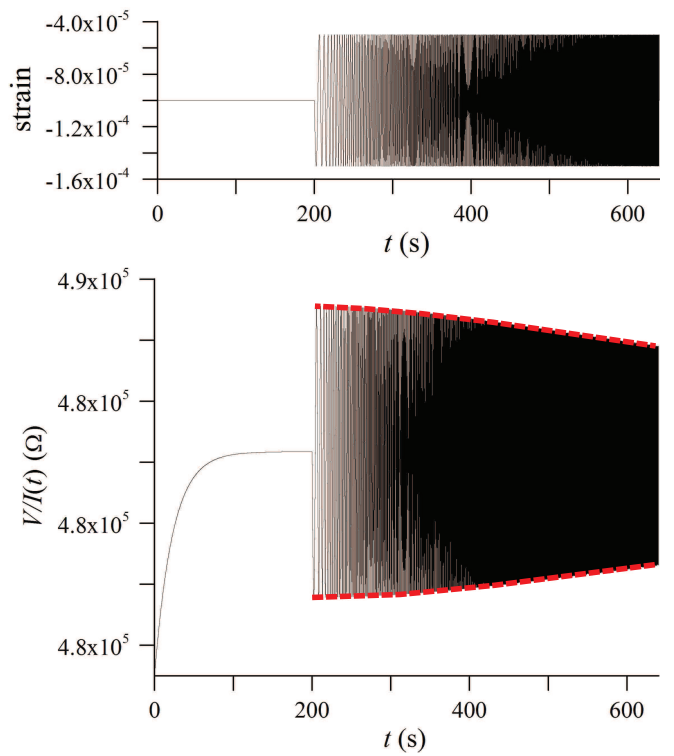

Figure 2: Sketch of the sensing unit commonly used in the literature for electromechanical piezoresistive modeling of CNTCS (a), and numerical test showing apparent electrical resistance with forward sweep of the frequency from 0.1 to $3.0 \mathrm{~Hz}$ (b).

When a mechanical strain $\epsilon$ (negative in compression) is applied to the sensor, the distance between the selected electrodes varies from $d_{o}$ to $d_{o}(1+\epsilon)$ and, consequently, $R_{i p}$ and $C_{i p}$ vary, while $R_{c t}$ remains approximately constant. In this first stage, we hypothesize that only the internal resistance is influenced by the mechanical deformation, so that the resistance changes as $R_{i p}=R_{i p}^{o}+\Delta R_{i p}$, where $\Delta R_{i p}$ represents the incremental change. In addition, the relationship between the relative change in the internal electrical resistance, $\Delta R_{i p} / R_{i p}^{o}$, and the axial strain, $\epsilon(t)$, is assumed to be linear:

$$
\frac{\Delta R_{i p}}{R_{i p}^{o}}=\lambda \epsilon(t)
$$

where $\lambda$ denotes the gauge factor of the sensor.

As illustrated in Fig. 1, the current passing through the sensor, $I(t)$, under a stabilized voltage, $V$, and axial strain, $\epsilon(t)$, is measured using a multimeter. From this model, the ordinary differential equation defining the time evolution of the current $I(t)$ is obtained by substituting Eq. (3) into Eq. (2), and after some rearranging of Eq. (1) one finds:

$$
\frac{\mathrm{d} I(t)}{\mathrm{d} t}+\frac{1}{\tau \bar{R}}\left(R_{i p}^{o}+\frac{R_{c t}}{1+\lambda \epsilon(t)}\right) I(t)=\frac{1}{1+\lambda \epsilon(t)} \frac{V}{\tau \bar{R}}
$$

Hence, the response of the sensor subjected to a harmonic axial deformation, $\epsilon(t)=-E \sin (w t)$, of amplitude $E$ and circular frequency $w$, is governed by the following linear differential equation with time-varying coefficients:

$$
\frac{\mathrm{d} I(t)}{\mathrm{d} t}+\frac{1}{\tau \bar{R}}\left(R_{i p}^{o}+\frac{R_{c t}}{1-\lambda E \sin (w t)}\right) I(t)=\frac{1}{1-\lambda E \sin (w t)} \frac{V}{\tau \bar{R}}
$$

The solution of this differential equation, in terms of apparent electrical resistance, $R_{a}(t)$, defined as the ratio between the imposed voltage and the measured current, can be approximated as [54]:

$$
R_{a}(t)=\frac{V}{I(t)} \approx R_{a}^{o}(t)+R_{a}^{t}(t)
$$

where $R_{a}^{o}(t)$ is a slowly varying component related to charge build-up in the capacitor, and $R_{a}^{t}(t)$ is a fast component associated with strain sensing. This latter quantity is generally written as:

$$
R_{a}^{o}(t)=R_{i p}^{o} \lambda E \sum_{n=1}^{\infty} a_{n} \sin \left(n w t+\phi_{n}\right)
$$

which equation describes a harmonic distortion with respect to the monochromatic input, thus suggesting that the response of the sensor is characterized by superharmonic components of coefficients $a_{n}$ and phase lags $\phi_{n}$. The presence of superharmonics is not related to nonlinearities, but only to the time-varying nature of the governing 
linear differential equation. This behavior complies with the first requirement expected for the modeling and, therefore, it is possible to fit the response of a CNTCS subjected to a cyclic loading as shown in [47, 50]. However, if Eq. (4) is solved for the case of an axial loading under a frequency sweep, the second requirement is not met. As can be seen in Fig. 2(b), the amplitude of the electrical resistance series decreases with increasing frequency of excitation and, consequently, the frequency response function (FRF) is a decreasing function which is in disagreement with experimental results presented in the literature.

\subsection{Piezoelectric equivalent circuit}

The reason for the amplification of the electrical signal with increasing frequency of excitation may be attributed to the formation of certain energy source. A similar behavior can be found with traditional piezoelectric materials, such as certain crystals and ceramics, in which an electric charge is accumulated in response to applied mechanical stress. To the best knowledge of the authors, all the published works to date have only associated the self-sensing capabilities of CNTCS to a piezoresistive behavior. However, since this behavior apparently does not explain the frequency response and the amplification of the electric signal under sweep frequency tests, the authors conducted experimental tests to determine whether some kind of piezoelectric effect can alter the dynamic response of the nanocomposites. Fig. 3 shows an example of the results obtained for this aim. In this case, a S1 type MWCNT/cement-paste sensor (posteriorly defined in Section 3) was subjected to external strains without power supply $(V=0 \mathrm{~V})$ using an universal testing machine. The voltage drop across the specimen was measured through a PXIe-4302 24-bit analog input module mounted on a PXIe-1071 chassis. Data from a load cell monitoring the compression force applied to the sensor were obtained using a 24-bit bridge input module (PXIe-4330). A correlation between the voltage and the applied loading can be observed. This confirms the coupling between the electric and the mechanical fields.

(a)
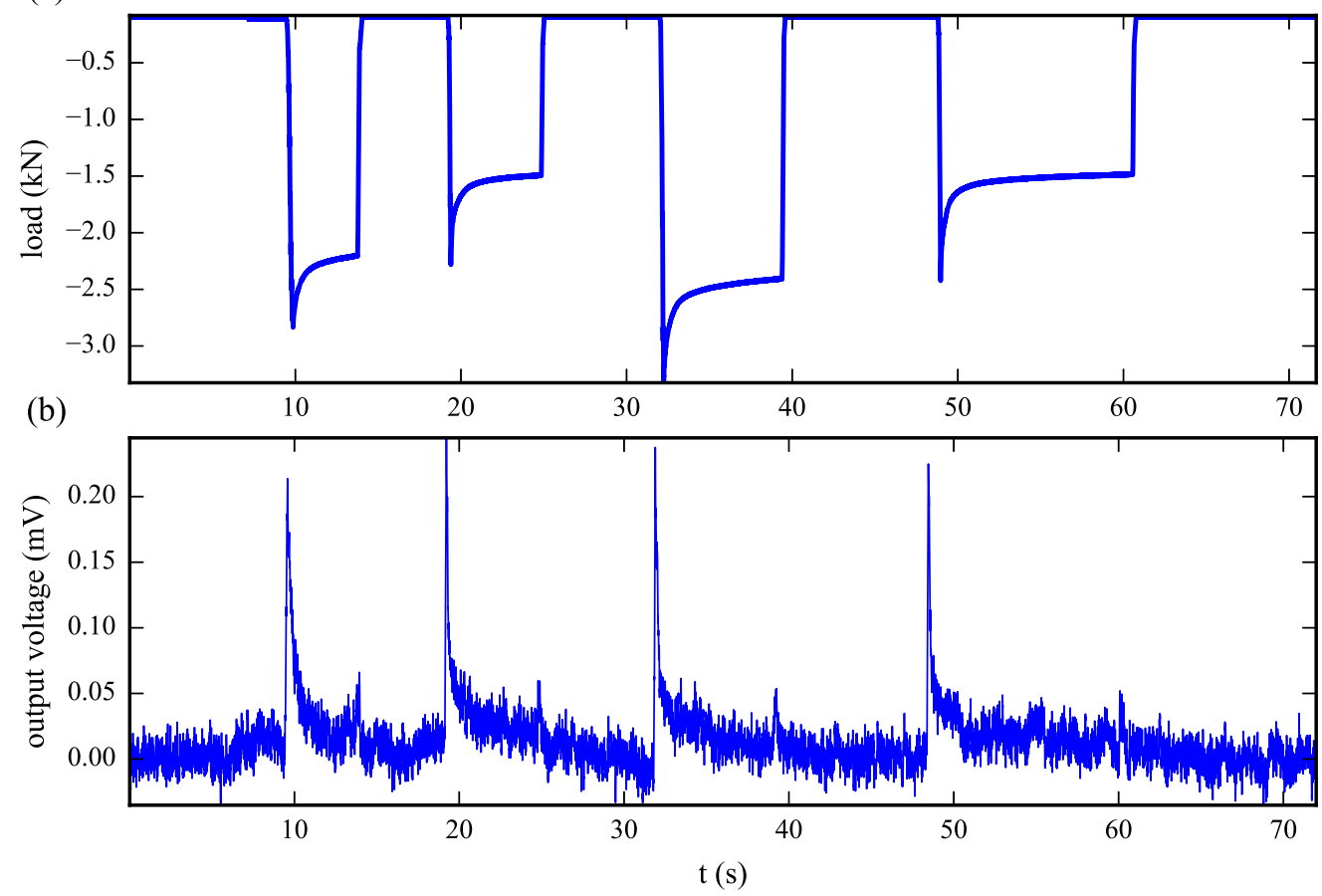

Figure 3: Time series of uni-axial compressive load acting on CNTCS without power supply (a), and output voltage (b) $(V=0$ $\mathrm{V}$, specimen S1).

In order to verify whether this piezoelectric behavior in combination with the previous model can explain the increasing frequency response function, a new sensing cell, as represented in Fig. 4(a), is analyzed. Assuming an ideal piezoelectric behavior, the charge generated by strain, $q$, can be related to the current introduced into the system $I_{p}$, as:

$$
I_{p}(t)=\frac{\mathrm{d} q(t)}{\mathrm{d} t}=S_{q} \frac{\mathrm{d} \epsilon(t)}{\mathrm{d} t}
$$

with $S_{q}$ being a piezoelectric parameter. Hence, Eq. (4) can be rewritten by simply applying Kirchoff's circuit laws as: 


$$
\frac{\mathrm{d} I(t)}{\mathrm{d} t}+\frac{1}{\tau \bar{R}}\left(R_{i p}^{o}+\frac{R_{c t}}{1+\lambda \epsilon(t)}\right) I(t)=\frac{1}{1+\lambda \epsilon(t)} \frac{V}{\tau \bar{R}}+\frac{S_{q}}{C_{i p} R_{c t}} \frac{\mathrm{d} \epsilon(t)}{\mathrm{d} t}
$$

The resulting differential equation with time-varying coefficients preserves a similar structure to Eq. (4), and the response under cyclic loadings also contains superharmonic components. However, in this case, the response under sweep frequency exhibits an amplification of the electrical signal with increasing frequency, as can be seen in Fig. 4(b).

(a)

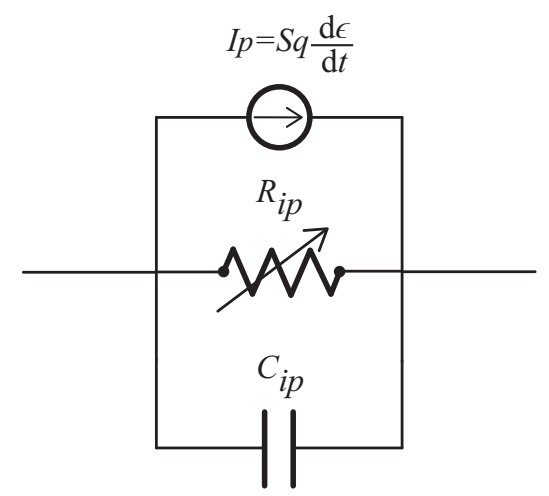

(b)
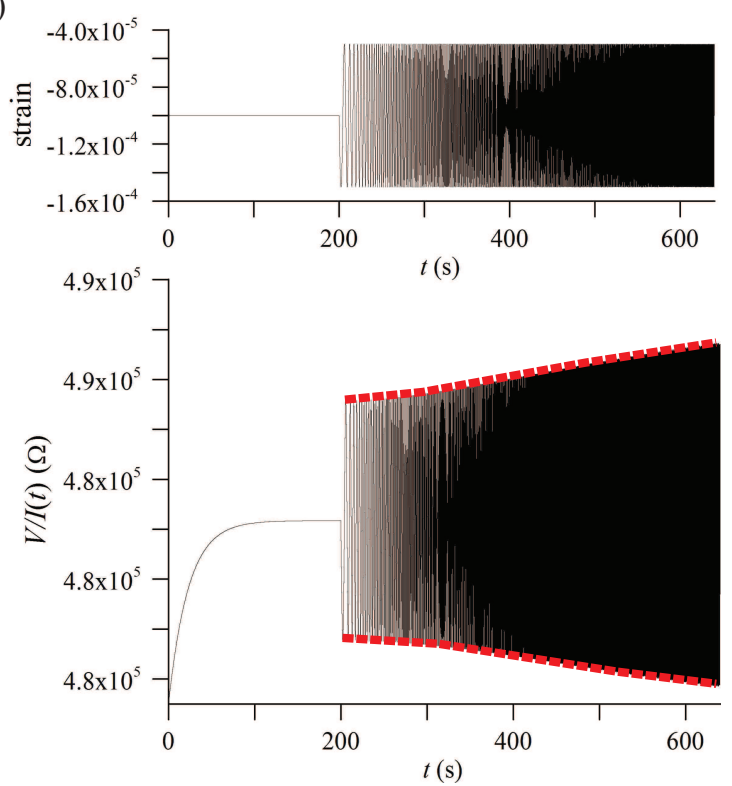

Figure 4: Sketch of the new sensing unit proposed for electromechanical piezoresistive modeling of CNTCS (a) and numerical test showing apparent electrical resistance with forward sweep of the frequency from 0.1 to $3.0 \mathrm{~Hz}(\mathrm{~b})$.

\subsection{Proposed equivalent electric circuit}

In the light of the experimental evidence of the piezoelectric response of CNTCS, a new lumped-circuit model is proposed to characterize its dynamic electromechanical behavior. The universal equivalent circuit model by Wansom et al. [53] is modified so as to include the piezoresistive effect associated with changes in the interparticle resistance following an applied mechanical strain, as well as the new piezoelectric effect. Regarding the piezoresistive effect, as adopted in the first model, many authors consider that only the internal resistance is influenced by the mechanical deformation. The origin of this assumption comes from the experimental results published by Han et al. [51]. However, there are also some other works defending the influence of the external strain on the internal capacitance, such as the work of Sanli et al. [52]. In that work, experimental results on (MWCNTs)/epoxy composites showed that, for small strains, there exists a linear relationship between the external strain and the internal capacitance. An explanation for this relationship is illustrated in Fig. 5. Each nanotube (or nanotube agglomerates) has a resistance $R_{C N T}$, and due to the inevitable coating of matrix material around the MWCNTs, there exists a dielectric gap which can be modeled as $R_{c}$ and $C_{\text {gap }}$ corresponding to the contact resistance and capacitance, respectively. Under external strains, there exists a certain change in resistance and capacitance of the network. This phenomenon can be explained as follows. The CNTs are randomly distributed within the matrix and they have a certain $R_{c}$ and $C_{g a p}$ values. Under the action of an external strain, the distance between CNTs is on average reduced, which will result in a decrease of $R_{c}$ and an increase in the $C_{g a p}$ value. Hence, for completeness, the strain-induced internal changes are assumed to be caused by variation of not only the internal resistance but also the internal capacitance. Therefore, a second gauge factor based on the relative capacitance change, $\lambda_{C}$, is defined as [52]:

$$
\lambda_{C}=-\frac{\Delta C / C_{o}}{\epsilon(t)}
$$

where $C_{o}$ is the unstrained capacitance and $\Delta C$ is the change in capacitance. 


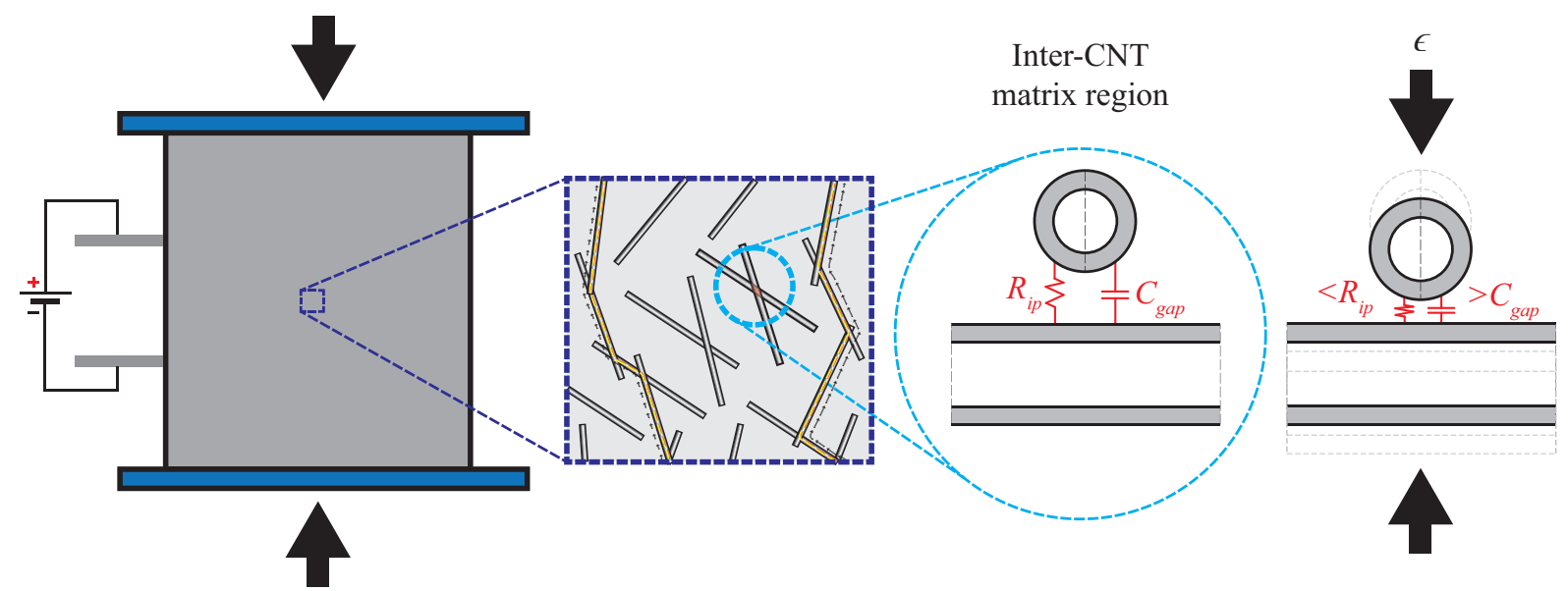

Figure 5: Schematic illustration at the micromechanical level of the equivalent RC model of the MWCNTs reinforced cementbased nanocomposite.

The proposed equivalent circuit is illustrated in Fig. 6(a). The sensing cell consists of two resistor-capacitor combinations (in parallel). The first branch is defined by $R_{i p}$ and $C_{i p}$ denoting the internal resistance and capacitance, respectively. It is followed by a second set of resistor-capacitor element defined by $R_{p m}$ and $C_{p m}$. Regarding the universal equivalent circuit proposed by Wansom et al. [53], the first resistor-capacitor set stands for the interparticle matrix path, in which the origin of piezoresistivity/piezoelectricity is hypothesized to take place, and the second one represents the interface response. For simplicity, the branches that represent the unreinforced matrix and the percolation path have been neglected as they presumably do not significantly contribute to the overall resistivity of the CNTCSs, because nanofiller content is assumed to be sufficiently smaller than the percolation threshold. Moreover, the resistor $R_{c t}$ encapsulates the contact resistances for cables, electrodes, and the specimens. In total, eight unique variables are required to characterize the behavior of the CNTCSs according to this model. In this paper, the proposed equivalent circuit was modeled using Simulink in MATLAB ${ }^{\circledR}$. The equivalent complex impedance $\left(Z_{e q}(w)\right)$ can be calculated as:

$$
Z_{e q}(\omega)=R_{c t}+\frac{1}{R_{i p}^{-1}+i \omega C_{i p}}+\frac{1}{R_{p m}^{-1}+i \omega C_{p m}}
$$

Fig. 6(b) shows a schematic of how the compression strain-induced changes lead to changes in CNTCS Nyquist impedance plot. The proposed model has an impedance response similar to previously published results (e.g. see [53]).
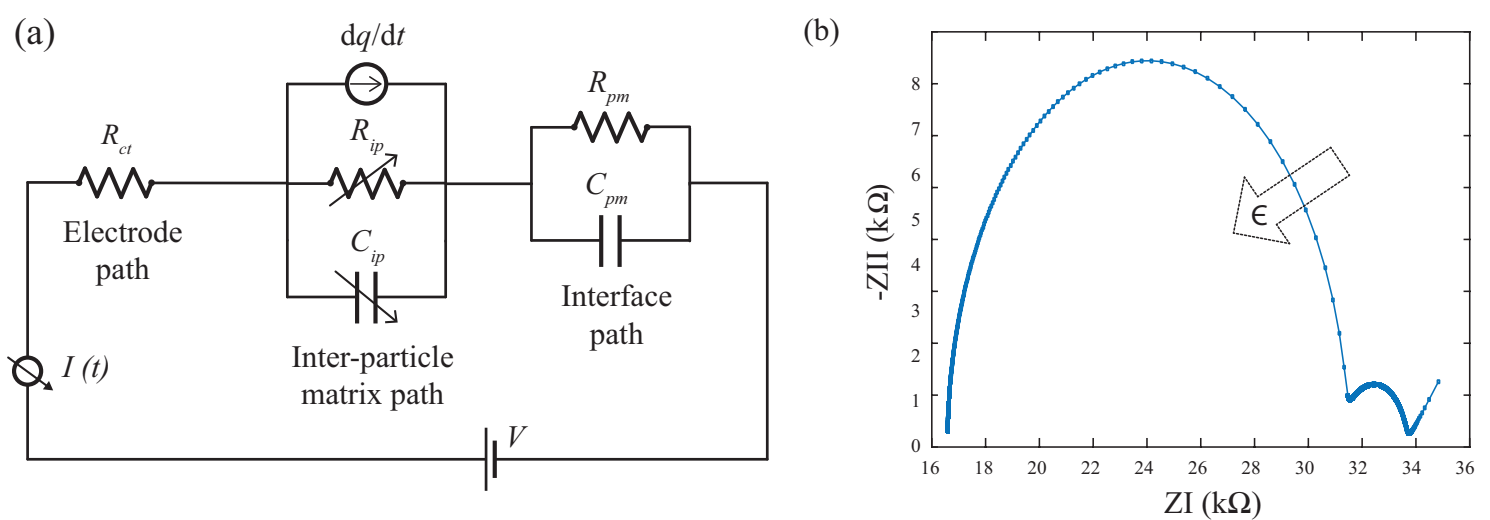

Figure 6: Equivalent electrical model (a) and simulated Nyquist diagram of CNTCS (b).

\section{Experimental methodology}

With the objective to validate the proposed model for the CNTCS, the model is used to reproduce the response of different CNTCS samples previously fabricated by the authors for material characterization and model validation. In particular, experimental data presented in [49] and [50] are here considered and a comparison with the previous model is conducted. The samples were made of cement paste doped with $2 \%$ of MWCNTs type Arkema 
Graphistrenth C100, with respect to the weight of cement. Cement-paste matrix was utilized because it provides more homogeneous materials in comparison with mortar or concrete. The nanofillers were dispersed in the cement matrix by 15 minutes of mechanical mixing and 15 minutes of sonication with a power of $225 \mathrm{~W}$. Two different types of prismatic specimens were used as shown in Fig. 7. The first type, (S1), has a cross-section of $40 \times 50$ $\mathrm{mm}^{2}$, and height of $50 \mathrm{~mm}$. The embedded electrodes are stainless steel nets. Specifically, the two inner electrodes are placed at a distance of $2 \mathrm{~cm}$, the external electrodes have a relative distance of $4 \mathrm{~cm}$, and the distance between an internal and an external electrode is $1 \mathrm{~cm}$. The second sample, (S2), has a square cross-section of $40 \times 40 \mathrm{~mm}^{2}$, and height of $160 \mathrm{~mm}$. In this case, the electrodes are copper wires with a diameter of $1 \mathrm{~mm}$, embedded in the middle of the sample. To avoid significant amplification phenomena within the working frequency range, the sensors were designed to have a natural vibration frequency similar to that of the acceleration transducers commonly used in civil engineering applications. The natural frequency of the sensors, $f_{o}$, was estimated using a well-known formula for the axial vibration of continuous rods:

$$
f_{o}=\frac{1}{2 L} \sqrt{\frac{E}{\rho}}
$$

where $L$ is the length of the sensor, $E$ is its Young's modulus and $\rho$ is the mass density. Eq. (12) is valid assuming a linearly elastic material, fixed axial displacements at both ends of the specimen and modeling the sensor as a monodimensional element. This assumption is a rough approximation of the natural frequency of the sensors which is useful for estimating at least its order of magnitude. In particular, assuming a tangent Young modulus equal to $7000 \mathrm{MPa}$, and using the measured value for $\rho$, equal to $1680 \mathrm{~kg} / \mathrm{m}^{3}$, Eq. (12) yields $f_{o}=6.4 \mathrm{kHz}$ in the case of $\mathrm{S} 1$ specimens.

Fig. 7 shows the configuration of the dynamic tests. Two teflon sheets provided insulation between the sample and the testing machine. The samples were instrumented with two strain gauges placed at mid-height on opposite lateral sides. The axial loads were applied with a hydraulic press, type MTS Model 243.40T. The tests were conducted applying sinusoidal compressive loads with both constant and sweep variation of frequencies. The electromechanical tests were carried out after a polarization of $1000 \mathrm{~s}$. The acquisition system of the load consisted of an MTS Force Transducer Model 661.23F-01. The measurements were sampled with a Spider8 device at a sampling frequency of $25 \mathrm{~Hz}$. During the tests, a stabilized voltage of $30 \mathrm{~V}$ was applied with an electrometer type Keithley, model 6517B, with resistivity test fixture, model 8009. The electrical current was acquired and then sampled at $13.5 \mathrm{~Hz}$.

Four electrodes were embedded in the samples with the purpose of allowing measurements in different configurations, namely two-probe and four-probe methods. In the case of four-probe measurements, a stabilized current source is applied to the outer electrodes, while inner electrodes are only used for measuring [14]. However, the scope of this work is limited to the two-probe method and therefore only the internal electrodes were used as schemed in Fig. 1. The reason for this choice is that the two-probe method permits use of higher voltages what results in a more accurate measurement and a higher evidence of electrical variations. In addition, the particular position of the internal electrodes, at the same height of the strain gauges, allowed us to correlate the strain condition with the electrical response of the CNTCS. Nevertheless, future developments of this work shall explore the applicability of the proposed lumped circuit to different test configurations, such as the four-probe method. 


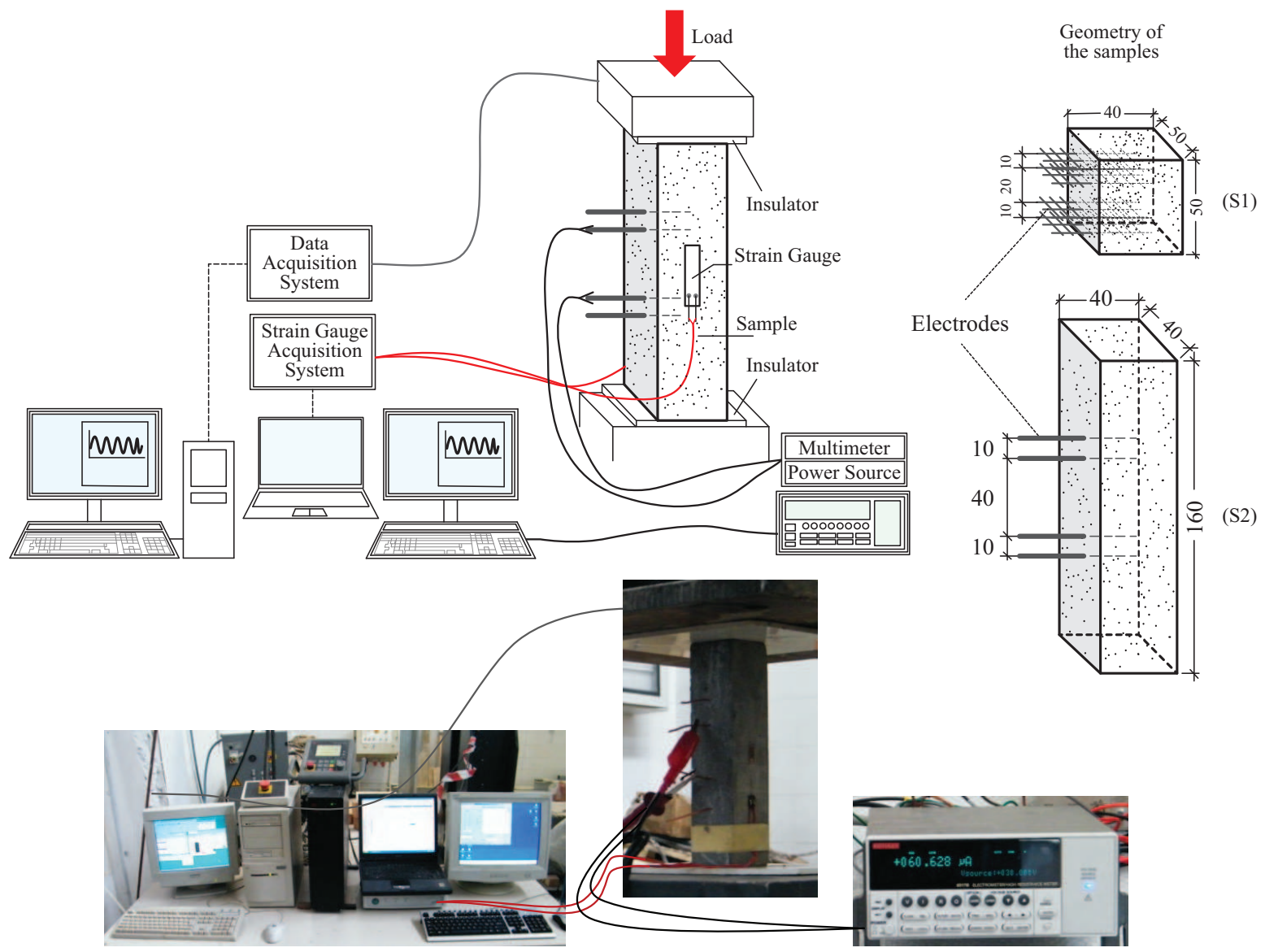

Figure 7: Setup of harmonic sinusoidal tests and geometry of the samples.

\section{Results and discussion}

The numerical tests presented in this section have been organized into three sections: step response under unloaded condition, harmonic excitation and frequency sweep analysis. At first, the analyses are used to verify the accuracy of the proposed equivalent circuit with comparison against experimental data. Furthermore, the tests are intended to demonstrate the capability of this approach to reproduce the two main features expected in the dynamic response of CNTCSs:

- The electric response of CNTCSs under harmonic excitations contains superharmonics.

- The frequency response function (FRF) of the sensor increases with the frequency of excitation.

\subsection{Step response of the unloaded specimen}

The objective of this first series of tests is to verify the validity of the proposed lumped electrical circuit model in the absence of mechanical deformations. The apparent electrical resistance is derived from the measured time history of the electrical current, $I(t)$, in response to a constant voltage difference, $V=1.5 \mathrm{~V}$, and taking $R(t)=V / I(t)$. The numerical solution is obtained by the Runge-Kutta method and a model-updating algorithm is implemented in order to fit the model parameters $\left\{R_{c t}, R_{i p}, R_{p m}, C_{i p}, C_{p m}, \lambda, \lambda_{C}, S_{q}\right\}$. The objective function seeks to minimize the least-squares difference between the numerical and the experimental results. In particular, $R_{c t}$ is directly obtained from the initial value of measured current intensity. The resulting parameters are presented in Table 1. In this case, the sensing capability is not activated, so there are not significant differences compared to previous piezoresistive modeling approaches. The accuracy of the model is verified by the quality of the fitting in Fig. 8. 


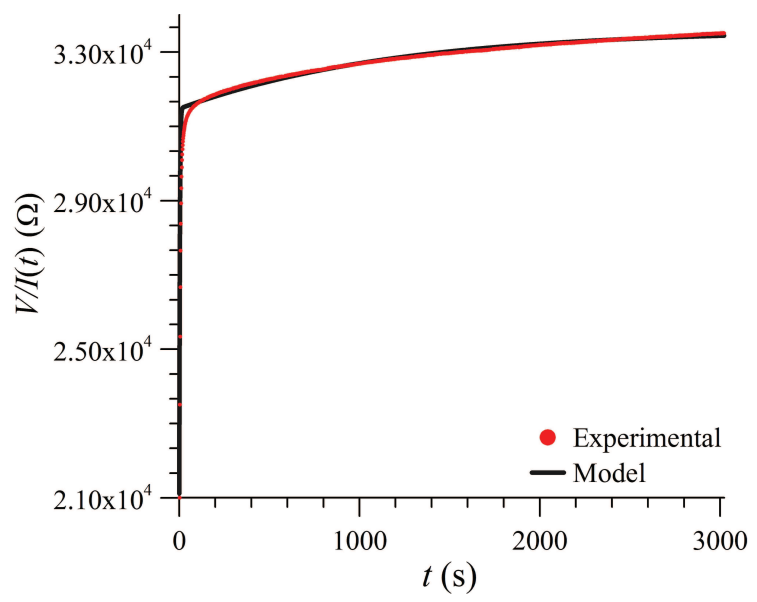

Figure 8: Comparison between experimental results and analytical predictions for the step response of CNTCS ( $V=1.5 \mathrm{~V}$, specimen $\mathrm{S} 1$ ).

Table 1: Fitted model parameters for the electrical step response of CNTCS ( $V=1.5 \mathrm{~V}$, specimen $\mathrm{S} 1)$.

\begin{tabular}{llllll}
\hline$R_{c t}(\mathrm{k} \Omega)$ & 21.12 & $C_{i p}(\mu \mathrm{F})$ & 385.70 & $\lambda$ & - \\
$R_{i p}(\mathrm{k} \Omega)$ & 10.36 & $C_{p m}(\mu \mathrm{F})$ & $5.59 \mathrm{E}+5$ & $\lambda_{C}$ & - \\
$R_{p m}(\mathrm{k} \Omega)$ & 2.12 & & & $S_{q}(\mathrm{~A})$ & - \\
\hline
\end{tabular}

\subsection{Cyclic loading}

The objective of the second set of tests is to assess the possibility of characterizing the strain sensitivity under harmonic excitations using the proposed circuit model. The experiments were conducted with the specimen S2 on different dates, and different room conditions. Therefore, the model parameters have been fitted to every test. In this particular case, two different frequencies of excitation, 0.1 and $0.2 \mathrm{~Hz}$, have been selected. Figs. 9 (a) and (b) show the full-length time histories of measured strain for the frequencies of $0.1 \mathrm{~Hz}$ and $0.2 \mathrm{~Hz}$, respectively. The tests were conducted after the application of a constant potential difference of $30 \mathrm{~V}$. A constant compression load of $2 \mathrm{kN}$ was applied for a duration of $240 \mathrm{~s}$ followed by sinusoidal loads with intensity varying from $2 \mathrm{kN}$ to $4 \mathrm{kN}$. The values of the applied stresses were chosen to investigate the electrical response of the sensors in the elastic uncracked state. The total duration of the tests was $360 \mathrm{~s}$. In this case, the updating algorithm has been defined as a multi-objective optimization problem to simultaneously fit both experiments. In addition, in order to highlight the improvements of the present circuit with respect to previously proposed approaches, the piezoresistive modeling shown in section 2.1 and documented in [50] is also analyzed. The fitted parameters for the newly proposed piezoresistive/piezoelectric model, A, and the previous piezoresistive model, B, are presented in Table 2. It is noted that in this test, as well as in all of the analyzed cases, the gauge factor based on the relative capacitance change, $\lambda_{C}$, has not shown a substantial effect on the response. A similar conclusion was reached by Han et al. [51] who experimentally showed that the compressive loading only causes a little insensitive and irregular change in capacitance of MWCNT/cement composites. The time traces of the electrical resistance measured in the experiments, as well as the ones predicted by models A and B, are shown in Fig. 10. It can be seen that the proposed equivalent circuit, model A, leads to accurate results for both frequencies of excitation. Conversely, model B which only considers the piezoresistive behavior is shown unable to reproduce the frequency-dependent amplitude of the electrical resistance. 
(a)

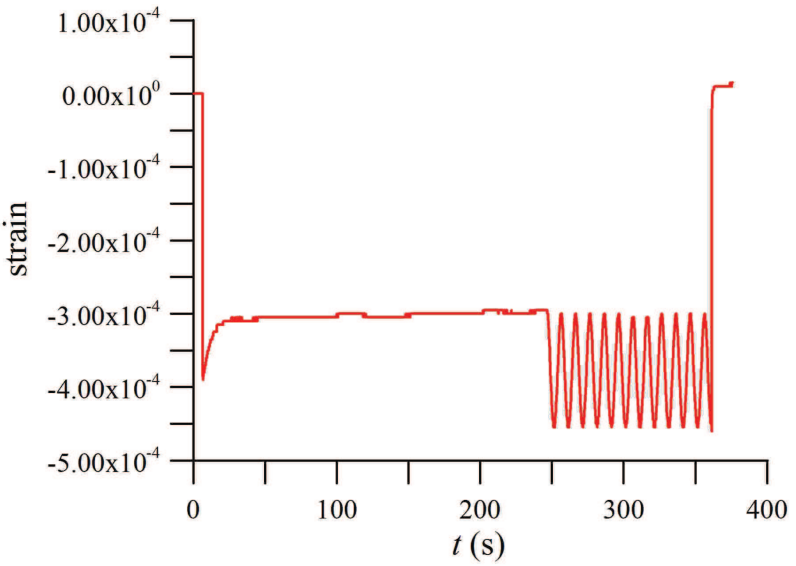

(b)

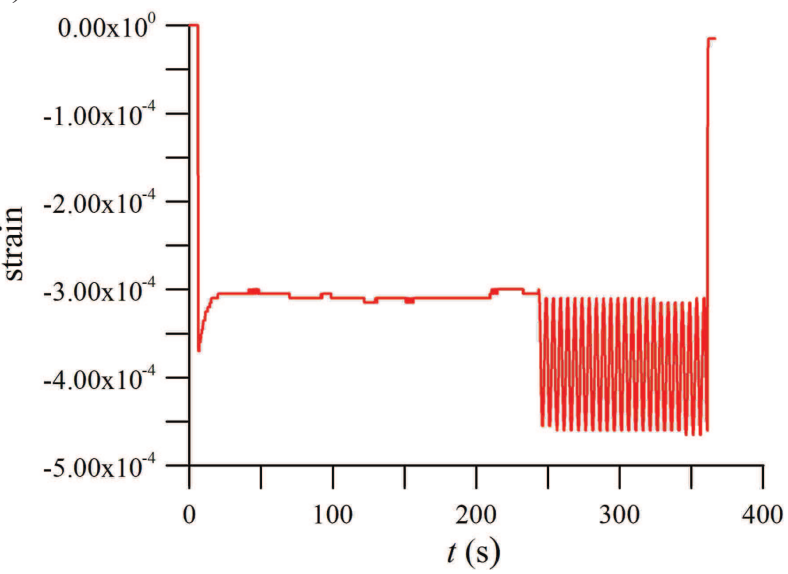

Figure 9: Strain series for cyclic loadings of $0.1 \mathrm{~Hz}(\mathrm{a})$ and $0.2 \mathrm{~Hz}(\mathrm{~b})(V=30 \mathrm{~V}$, specimen $\mathrm{S} 2)$.

(a)

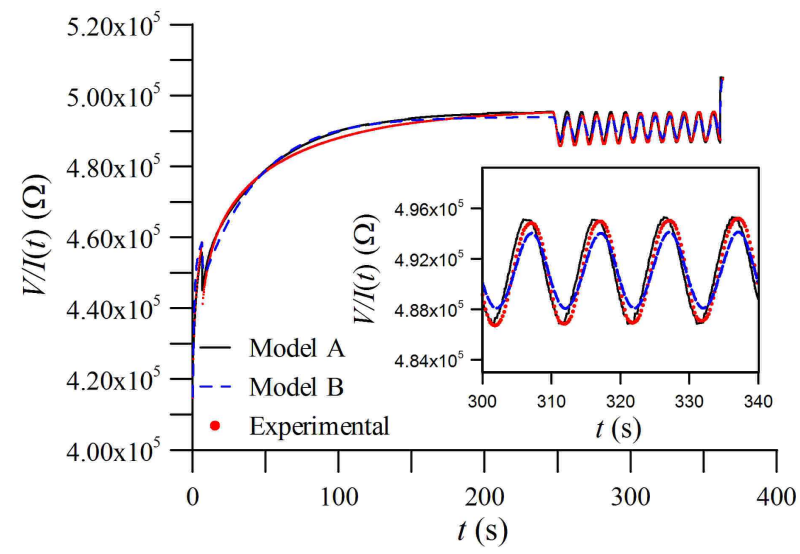

(b)

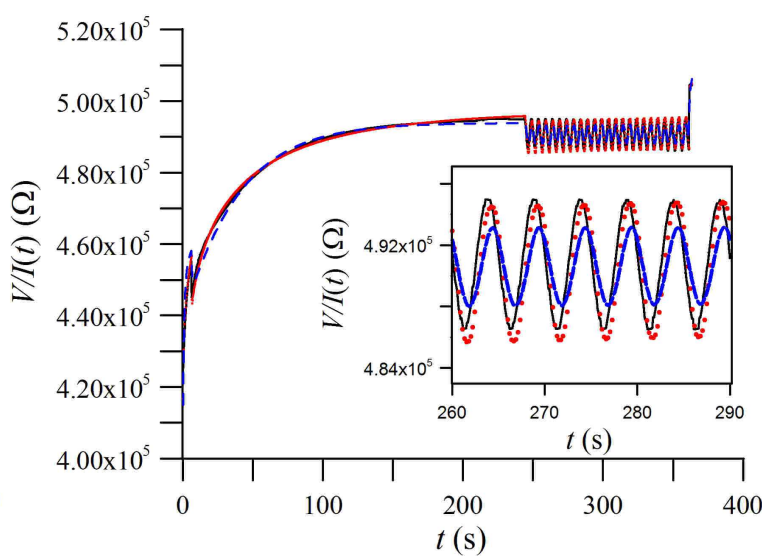

Figure 10: Comparison between experimental results and analytical predictions of the response of CNTCS for cyclic loadings of $0.1 \mathrm{~Hz}$ (a) and $0.2 \mathrm{~Hz}$ (b) $(V=30 \mathrm{~V}$, specimen S2). Models A and B correspond to the newly proposed piezoresistive/piezoelectric and previously published piezoresistive modeling, respectively.

Table 2: Fitted model parameters for cyclic loadings of $0.1 \mathrm{~Hz}$ and $0.2 \mathrm{~Hz}(V=30 \mathrm{~V}$, specimen S2). Models A and B correspond to the newly proposed piezoresistive/piezoelectric and previously published piezoresistive modeling, respectively.

\begin{tabular}{|c|c|c|c|c|c|c|c|c|c|c|c|}
\hline \multicolumn{6}{|c|}{ Model A (piezoresistive/piezoelectric) } & \multicolumn{6}{|c|}{ Model B (piezoresistive) } \\
\hline$R_{c t}(\mathrm{k} \Omega)$ & 200.00 & $C_{i p}(\mu \mathrm{F})$ & 300.00 & $\lambda$ & 300.00 & $R_{c t}(\mathrm{k} \Omega)$ & 404.01 & $C_{i p}(\mu \mathrm{F})$ & 19.98 & $\lambda$ & 802.55 \\
\hline $\begin{array}{l}R_{i p}(\mathrm{k} \Omega) \\
R_{p m}(\mathrm{k} \Omega)\end{array}$ & $\begin{array}{l}289.50 \\
50.00\end{array}$ & $C_{p m}(\mu \mathrm{F})$ & $2.00 \mathrm{E}+04$ & $\begin{array}{l}\lambda_{C} \\
S_{q}(\mathrm{~A})\end{array}$ & $\begin{array}{l}2.21 \mathrm{E}-09 \\
-0.40\end{array}$ & $\begin{array}{l}R_{i p}(\mathrm{k} \Omega) \\
R_{p m}(\mathrm{k} \Omega)\end{array}$ & $\begin{array}{l}46.41 \\
54.84\end{array}$ & $C_{p m}(\mu \mathrm{F})$ & 745.78 & $\begin{array}{l}\lambda_{C} \\
S_{q}(\mathrm{~A})\end{array}$ & $\begin{array}{l}3.08 \mathrm{E}-14 \\
-\end{array}$ \\
\hline
\end{tabular}

In order to gain a better insight into the potential of the studied equivalent circuit, the electrical response in the frequency domain is analyzed. In this case, similarly to the latter tests, a frequency of excitation of $2 \mathrm{~Hz}$ is selected. The fitted parameters for models A and B are presented in Table 3. It is worth noting that parameters of model A are similar in Tables 2 and 3, even though some differences are evidenced, that are conceivably determined by different testing conditions. On the contrary, parameters of model B as identified in Tables 2 and 3 show large differences, what most likely indicates that modeling errors are more significant in this case. Figs. 11(a), (b) and (c) show the time history of the apparent electrical resistance obtained in the experiment, by model A, and model $\mathrm{B}$, respectively. The slowly varying component, $R_{a}^{o}(t)$, associated with the polarization of cement-based materials, has been eliminated with a high-pass filter with a cutoff frequency of $0.2 \mathrm{~Hz}$. Fig. 11(d) shows the power spectral density (PSD) of these three signals. In agreement with Eq. (7), the results reported therein demonstrate that the dynamic response of the sensor is not monochromatic, but rather contains superharmonics at $4 \mathrm{~Hz}, 6 \mathrm{~Hz}$, and other multiples of $2 \mathrm{~Hz}$. Both methodologies can reproduce the superharmonic content of the electrical signals 
with fairly good agreements with the experimental data. This explains why previous piezoresistive modelings have been shown able to reproduce the response of CNTCSs under the action of one single value of harmonic loading.

(a)

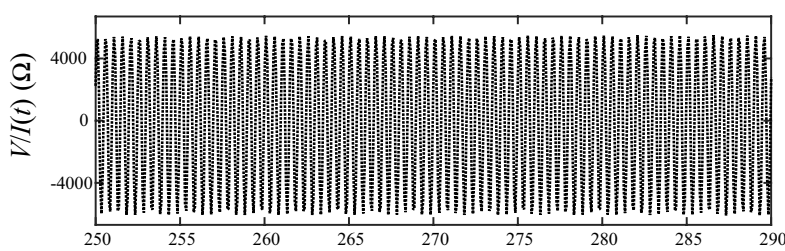

(b)

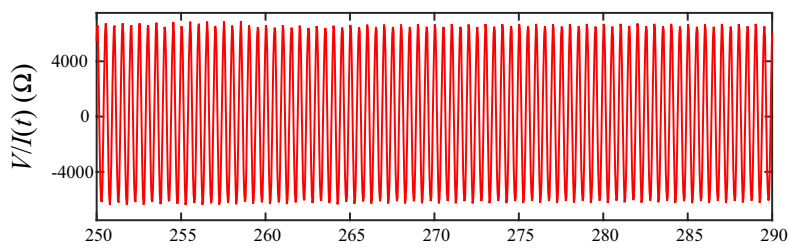

(c)

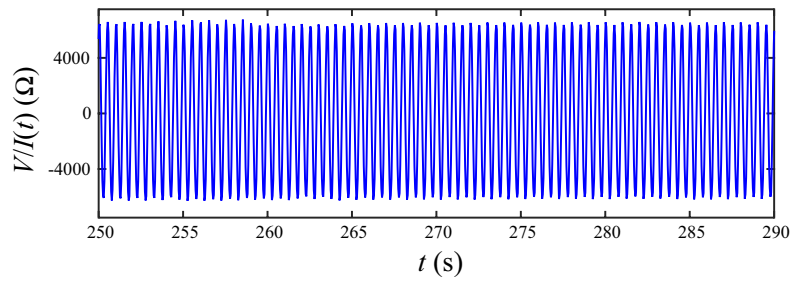

(d)

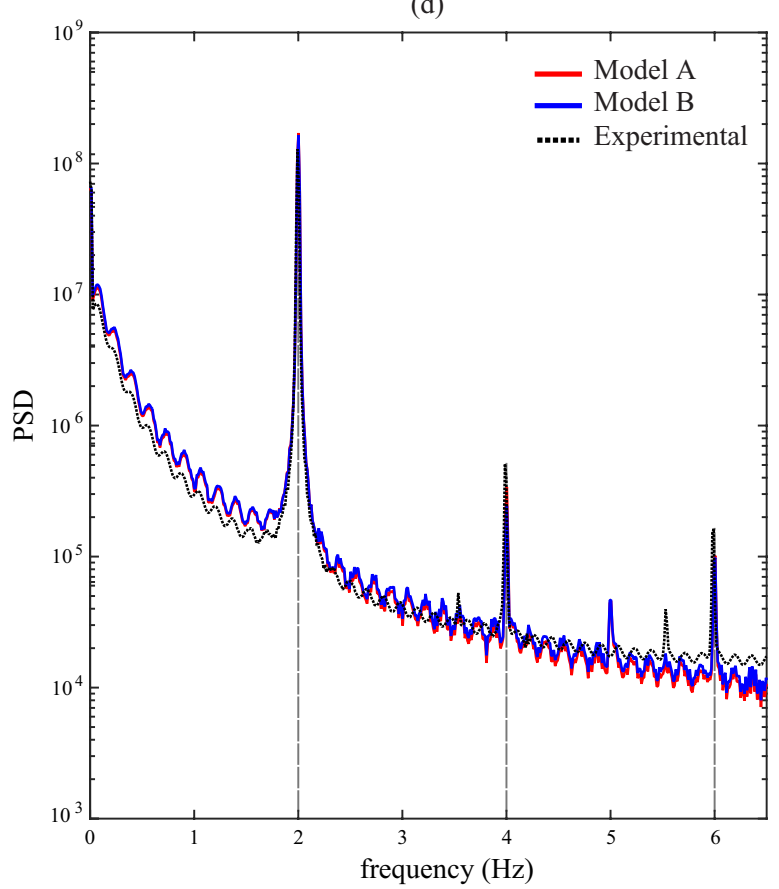

Figure 11: Variation of the apparent resistance obtained in experiments (a), model A (b) and model B (c) and their PSDs (d) under $5 \mathrm{~Hz}$ sinusoidal axial loading ( $V=30 \mathrm{~V}$, specimen S2). Models A and B correspond to the newly proposed piezoresistive/piezoelectric and previously published piezoresistive modeling, respectively.

Table 3: Fitted model parameters for cyclic loading of $2 \mathrm{~Hz}$ ( $V=30 \mathrm{~V}$, specimen S2). Models A and B correspond to the newly proposed piezoresistive/piezoelectric and previously published piezoresistive modeling, respectively.

\begin{tabular}{|c|c|c|c|c|c|c|c|c|c|c|c|}
\hline \multicolumn{6}{|c|}{ Model A (piezoresistive/piezoelectric) } & \multicolumn{6}{|c|}{ Model B (piezoresistive) } \\
\hline$R_{c t}(\mathrm{k} \Omega)$ & 129.74 & $C_{i p}(\mu \mathrm{F})$ & 285.00 & $\lambda$ & 300.00 & $R_{c t}(\mathrm{k} \Omega)$ & 13.20 & $C_{i p}(\mu \mathrm{F})$ & 0.00 & $\lambda$ & 196.00 \\
\hline$R_{i p}(\mathrm{k} \Omega)$ & 313.53 & $C_{p m}(\mu \mathrm{F})$ & $2.13 \mathrm{E}+04$ & $\lambda_{C}$ & 0.00 & $R_{i p}(\mathrm{k} \Omega)$ & 320.30 & $C_{p m}(\mu \mathrm{F})$ & $1.49 \mathrm{E}+05$ & $\lambda_{C}$ & 0.00 \\
\hline$R_{p m}(\mathrm{k} \Omega)$ & 49.74 & & & $S_{q}(\mathrm{~A})$ & -0.50 & $R_{p m}(\mathrm{k} \Omega)$ & 12913.00 & & & $S_{q}(\mathrm{~A})$ & - \\
\hline
\end{tabular}

\subsection{Frequency sweep analysis}

Having shown the capability of the proposed equivalent circuit in reproducing the superharmonics in the dynamic response of CNTCSs under cyclic loadings, a sweep test has conducted to investigate the response under varying frequency of excitation. The tests consisted of the initial application of a constant compression load of $2 \mathrm{kN}$ for $70 \mathrm{~s}$ followed by concatenated sinusoidal compression loads with different frequencies and amplitudes varying from $2 \mathrm{kN}$ to $4 \mathrm{kN}$. In total, 10 load cycles were applied at 13 different load frequency values: from 0.1 to $0.5 \mathrm{~Hz}$ with step increments of $0.1 \mathrm{~Hz}, 0.75 \mathrm{~Hz}$, and from 1.0 to $3.5 \mathrm{~Hz}$ with step increments of $0.5 \mathrm{~Hz}$. The total duration of the test was approximately $340 \mathrm{~s}$. The fitted parameters for models A and B are shown in Table 4. Note the fitted parameters for the newly proposed model A, unlike model B, are still consistent with the previous results in Tables 2 and 3. Fig. 12(a) shows the time series of measured strain. Fig. 12(b) shows the comparison of the experimental results and the fitted numerical results obtained by models A and B. As mentioned above, the amplitude of the relative electrical resistance is frequency-dependent. In particular, the amplitude has been shown to be increasing with the frequency. Model B, which only considers piezoresistive effects, exhibits the opposite behavior. On the contrary, the proposed approach, model A, does reproduce a direct frequencydependent amplitude of the electrical resistance leading to a very good agreement with the experimental data. The proposed approach for a forward and backward frequency sweep analysis is shown in Fig. 13. The slowly varying component has been removed by a high-pass filter and the mean value has been extracted. The envelope of the signal was extracted with the amplitude of the analytical signal obtained by a Hilbert transform. Hence, it can be seen that this model provides electrical resistance amplitude increasing with the frequency of excitation for both forward and backward frequency sweep analyses. 
(a)

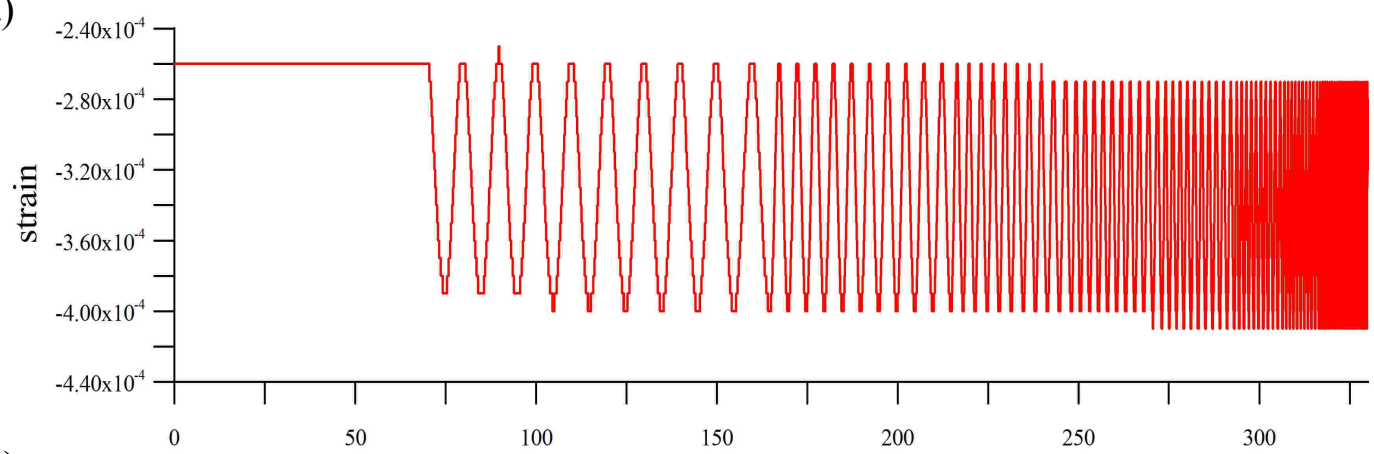

(b)

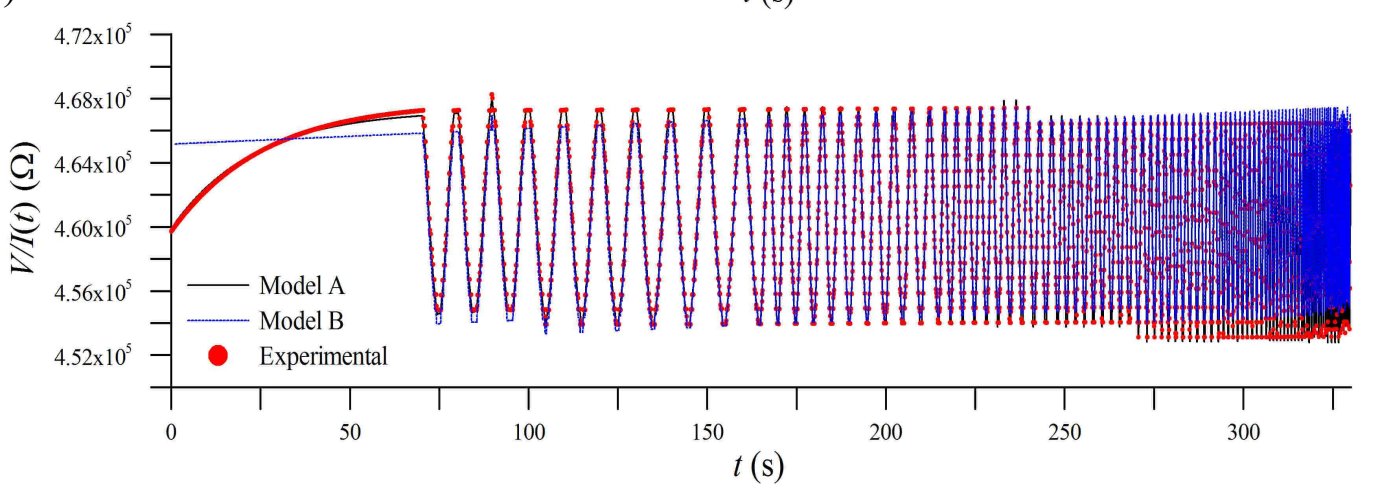

Figure 12: Strain series (a) and comparison between experimental results and analytical predictions of the response of CNTCS for forward sweep test from 0.1 to $3.5 \mathrm{~Hz}$ (b) $(V=30 \mathrm{~V}$, specimen S2). Models A and B correspond to the newly proposed piezoresistive/piezoelectric and previously published piezoresistive modeling, respectively.

Table 4: Fitted model parameters for sweep test from 0.1 to $3.5 \mathrm{~Hz}$ ( $V=30 \mathrm{~V}$, specimen S2). Models A and B correspond to the newly proposed piezoresistive/piezoelectric and previously published piezoresistive modeling, respectively.

\begin{tabular}{|c|c|c|c|c|c|c|c|c|c|c|c|}
\hline \multicolumn{6}{|c|}{ Model A (piezoresistive/piezoelectric) } & \multicolumn{6}{|c|}{ Model B (piezoresistive) } \\
\hline$R_{c t}(\mathrm{k} \Omega)$ & 129.85 & $C_{i p}(\mu \mathrm{F})$ & 284.75 & $\lambda$ & 300.00 & $R_{c t}(\mathrm{k} \Omega)$ & 13.31 & $C_{i p}(\mu \mathrm{F})$ & 0.00 & $\lambda$ & 195.20 \\
\hline$R_{i p}(\mathrm{k} \Omega)$ & 313.42 & $C_{p m}(\mu \mathrm{F})$ & $2.13 \mathrm{E}+04$ & $\lambda_{C}$ & 0.00 & $R_{i p}(\mathrm{k} \Omega)$ & 320.16 & $C_{p m}(\mu \mathrm{F})$ & $1.49 \mathrm{E}+05$ & $\lambda_{C}$ & 0.00 \\
\hline$R_{p m}(\mathrm{k} \Omega)$ & 49.74 & & & $S_{q}(\mathrm{~A})$ & -0.50 & $R_{p m}(\mathrm{k} \Omega)$ & 12913.00 & & & $S_{q}(\mathrm{~A})$ & - \\
\hline
\end{tabular}


(a)

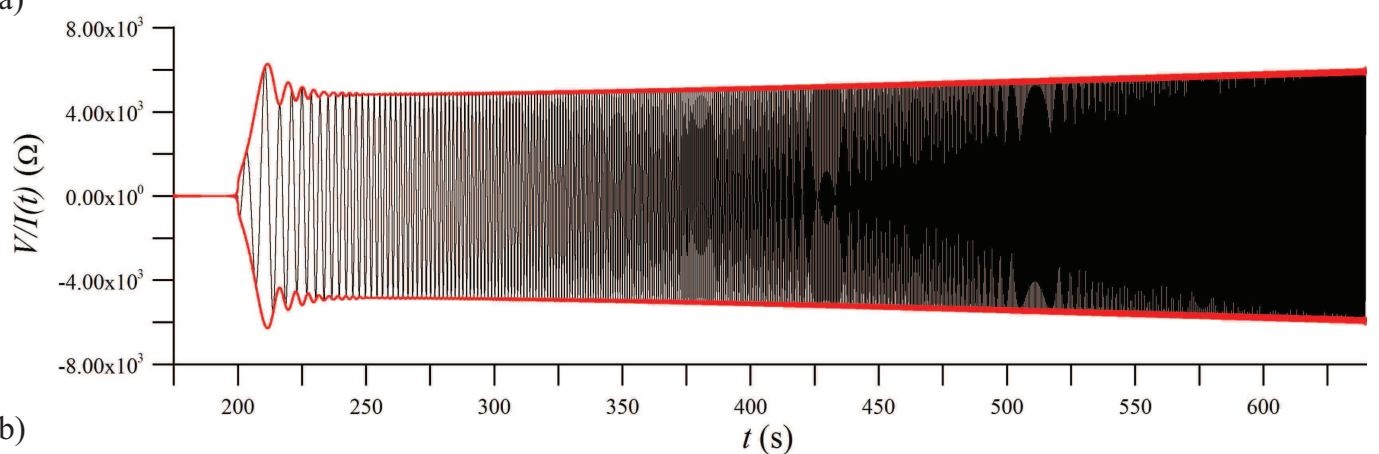

(b)

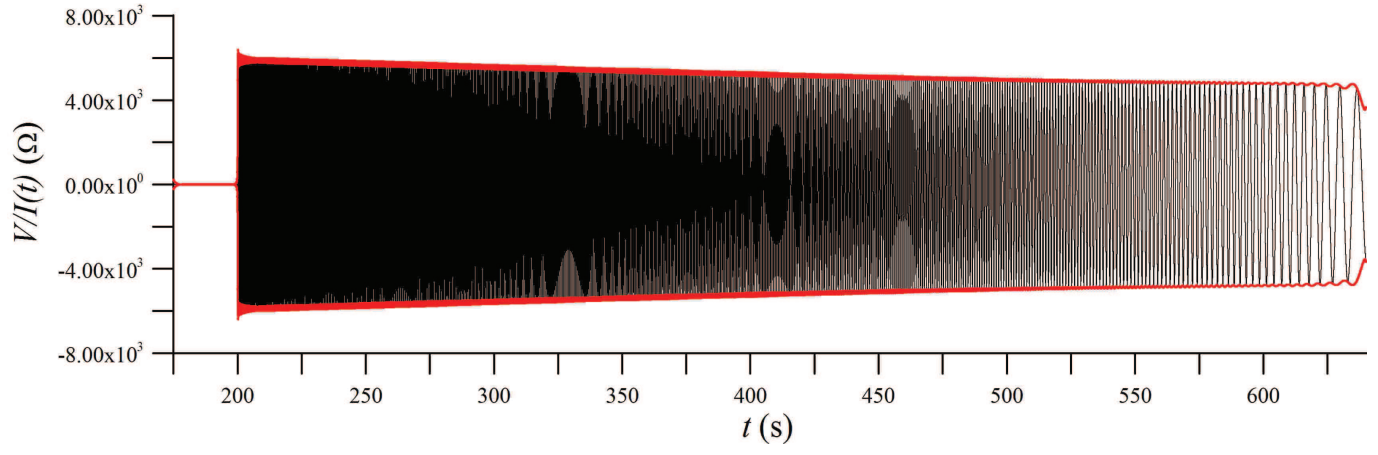

Figure 13: Analytical predictions of the response of CNTCS for forward (a) and backward (b) sweep test from 0.1 to $3.5 \mathrm{~Hz}$ obtained by the newly proposed piezoresistive/piezoelectric modeling ( $V=30 \mathrm{~V}$, specimen $\mathrm{S} 2)$.

The response of a dynamic transducer governed by a linear input-output differential equation is fully described by its frequency response function. The frequency response function is a transfer function that describes the system response to an external excitation force as a function of frequency as:

$$
\operatorname{FRF}(f)=\frac{Y(f)}{X(f)}
$$

where $Y(f)$ is the Fourier transform of the output response $y(t), X(f)$ is the Fourier transform of the input $x(t)$, and $f$ denotes frequency. The dynamic response of transducers commonly used for measuring vibrations is generally described by a second order differential input-output equation. The FRF of this type of systems only depends on the resonant frequency of the sensor and its damping. When the excitation frequency remains far below the resonant frequency of the sensor, $f_{o}$, as it is the case, the FRF can be approximated with a constant function with zero phase. This approach corresponds to the response of an ideal linear input-output transducer without delay. In this situation, the level of approximation of a constant FRF is essentially independent of the damping.

The results of the sweep tests have been used to characterize the amplitude of the FRF. To this end, the oscillating components have been first obtained by removing the best-fitting linear trend from the full-length records after the initial $70 \mathrm{~s}$. Next, time windows with 10 cycles at constant frequencies have been extracted, and the average peak-to-peak amplitude of both electrical resistance and the applied load has been evaluated. Finally, the FRF has been obtained using Eq. (13), and the resulting curve has been normalized by its value corresponding to the lowest frequency $(0.1 \mathrm{~Hz})$. The results, obtained for both the forward and backward frequency sweep tests, are shown in Fig. 14(a). Only the interval 0.1-3.5 Hz is shown because sampling errors became unacceptable for higher frequencies. For comparative purposes, Fig. 14(a) also shows the mechanical FRF that would result from the ideal behavior of the sensors modeled as second-order systems with natural frequency equal to $f_{o}$. A critical damping ratio of 0.01 has been considered as representative of the internal damping of hardened cement paste in uncracked state [55]. The experimental results in Fig. 14(a) confirm the prediction of a slight amplitude increase of the response with the frequency. It must be noted that the comparison between the FRFs extracted from forward and backward frequency sweep responses shows a close match what indicates that the errors associated with the elimination of the trend from the time histories and to the sampling were limited in this range.

The FRF has been similarly obtained with the newly proposed equivalent circuit and represented in Fig. 14(a). The model parameters have been taken from the frequency sweep analysis in Table 4 . It can be seen that the solution with the fitted piezoelectric parameter in the frequency sweep analysis, $S_{q}=-0.50$ A, provides a reasonably good agreement with the experimental data. The FRF has been also obtained with the proposed equivalent circuit for different values of piezoelectric parameter, $S_{q}$, as shown in Fig. 14(b). It is noted that the amplification of the 
electric resistance with frequency can be modulated with different piezoelectric parameters, that is, higher values of $S_{q}$ lead to higher increasing FRFs. The case of $S_{q}=0$, corresponding to the case of only piezoresistive model and equivalent to model B, provides decreasing FRFs with excitation frequency. These results demonstrate that the proposed equivalent circuit is superior in comparison to the previous one in reproducing the dynamic response of the sensors. Moreover, this modeling confirms that the weakly non-linear FRF is not due to mechanical reasons but rather to the peculiar eletrodynamic behavior of the cement paste prepared with carbon nanotubes in this study.

(a)

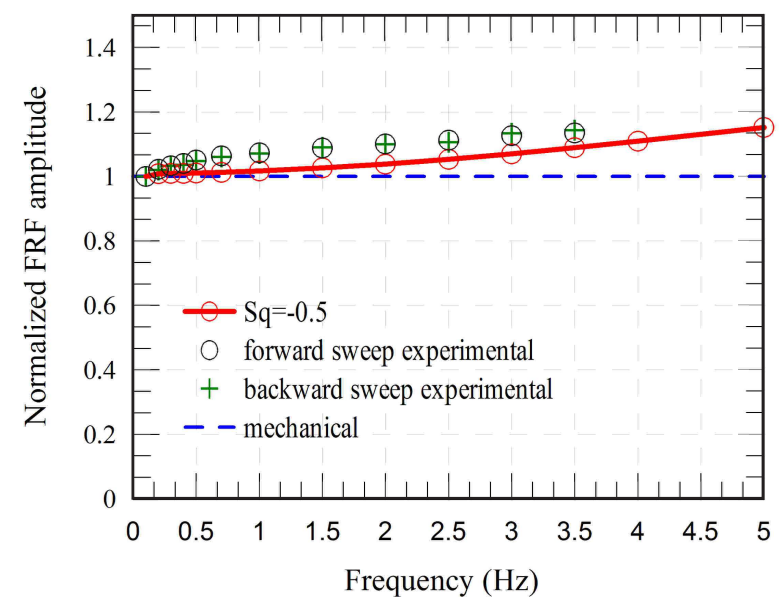

(b)

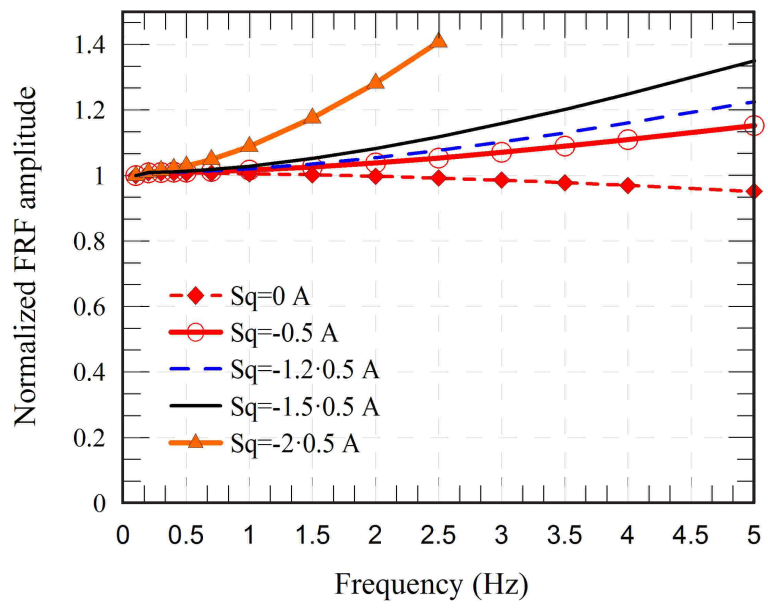

Figure 14: Comparison of the experimental and numerical frequency response functions (FRFs) of CNTCSs (a), and sensitivity analysis of the piezoelectric parameter $S_{q}(V=30 \mathrm{~V}$, specimen $\mathrm{S} 2)$.

\section{Conclusions}

In this paper, we have presented an electromechanical model for CNTCSs based on an equivalent lumped circuit. The model consists of a resistor in series with two RC elements, standing for the contact resistance, inter-particle matrix path, and the interface response, respectively. On the basis of experimental evidence, this paper has proposed a novel piezoelectric/piezoresistive approach for the dynamic modeling of CNTCSs. The piezoresistive effect is simulated by a linear variation of the bulk resistance with strain, and the piezoelectric effect is modeled as an additional current source originated by external loadings. Experimental tests of step response of unloaded specimens, harmonic excitations and frequency sweep tests have been performed as a benchmark for validation. The parameters have been fitted by least-square differences optimization and excellent agreements have been found with experimental data. The results have demonstrated that the proposed model can reproduce the superharmonic response of CNTCSs under cyclic loadings, as well as the amplification of the electric resistance with the frequency of excitation.

A series of experiments have been conducted to verify the equivalent circuit model adopted for CNTCSs. To investigate the electrical behavior of the CNTCS under applied mechanical deformations, the samples were connected to a power source providing a stabilized potential difference across their electrodes, and to a digital multimeter measuring the time history of the current passing through the specimen. Dynamic characterization was conducted using a servo-controlled pneumatic universal testing machine equipped with an environmental chamber to control for constant temperature. The applied axial deformation was measured through two off-theshelf resistive strain gauges attached to the opposite lateral faces of the specimen. Three different sets of tests have been performed including step response under unloaded conditions, harmonic excitations and sweep analyses.

The main contributions of this paper are summarized below:

- Experimental results evidenced that mechanical deformations in the absence of externally applied power source generate voltage impulses between the electrodes of the specimen. This fact demonstrates the existence of certain level of piezoelectricity in cement-based materials doped with CNTs.

- The proposed model can accurately reproduce the step response of CNTCSs under unloaded conditions.

- The electrical response of CNTCSs in terms of electrical resistance under cyclic loadings contains superharmonics. Both piezoresistive and piezoelectric/piezoresistive modelings can reproduce this behavior.

- The variation of the internal capacitance with external strain has been shown to be negligible. 
- The amplitude of the electric resistance is increasing with the frequency of excitation. Approaches that only take into account the piezoresistive effect have shown an opposite behavior, leading to decreasing amplitudes with increasing frequencies of excitation. On the contrary, the proposed piezoresistive/piezoelectric modeling has shown able to reproduce this effect and accurate fittings have been obtained for sweep analyses.

- The fitted parameters have been shown consistent under different experimental conditions and, therefore, the proposed model has proved capable of reproducing the internal behavior of CNTCSs.

- The amplification of the electrical resistance can be modulated with the piezoelectric parameter.

The present equivalent circuit is envisioned to provide a useful tool for signal processing applications of CNTCs in the realm of SHM. Further research work shall address different experimental configurations, such as the four-probe method, as well as the coupling with micromechanical approaches in order to predict the value of the parameters governing the equivalent lumped circuit.

\section{Acknowledgement}

This work was partially financed by the Ministerio de Economía y Competitividad of Spain under the project DPI2014-53947-R. E. G-M was also supported by a FPU contract-fellowship from the Spanish Ministry of Education Ref: FPU13/04892. The support of the Italian Ministry of Education, University and Research (MIUR) through the funded Project of Relevant National Interest (PRIN) entitled "SMART-BRICK: Novel strain-sensing nano-composite clay brick enabling self-monitoring masonry structures" (protocol no. 2015MS5L27) is gratefully acknowledged. This work is also partly supported by the National Science Foundation Grant No. 1069283, which supports the activities of the Integrative Graduate Education and Research Traineeship (IGERT) in Wind Energy Science, Engineering and Policy (WESEP) at Iowa State University.

\section{References}

[1] P.-C. Aïtcin, Cements of yesterday and today: concrete of tomorrow, Cement and Concrete research 30 (2000) 1349-1359.

[2] B. Mather, Concrete durability, Cement and Concrete Composites 26 (2004) 3-4.

[3] B. Han, J. Ou, Embedded piezoresistive cement-based stress/strain sensor, Sensors and Actuators A: Physical 138 (2007) 294-298.

[4] B. Han, X. Yu, E. Kwon, A self-sensing carbon nanotube/cement composite for traffic monitoring, Nanotechnology 20 (2009) 445501.

[5] F. Ubertini, A. L. Materazzi, A. D’Alessandro, S. Laflamme, Natural frequencies identification of a reinforced concrete beam using carbon nanotube cement-based sensors, Engineering Structures 60 (2014) $265-275$.

[6] A. D. B. Ferreira, P. R. Nóvoa, A. T. Marques, Multifunctional material systems: A state-of-the-art review, Composite Structures (2016).

[7] O. Galao, F. Baeza, E. Zornoza, P. Garcés, Strain and damage sensing properties on multifunctional cement composites with CNF admixture, Cement and concrete composites 46 (2014) 90-98.

[8] S. Laflamme, F. Ubertini, H. Saleem, A. D’Alessandro, A. Downey, H. Ceylan, A. L. Materazzi, Dynamic characterization of a soft elastomeric capacitor for structural health monitoring, Journal of Structural Engineering 141 (2014) 04014186.

[9] F. Ubertini, S. Laflamme, A. D’Alessandro, Smart cement paste with carbon nanotubes, Innovative Developments of Advanced Multifunctional Nanocomposites in Civil and Structural Engineering (2016) 97-120.

[10] H. Li, H. Xiao, J. Ou, A study on mechanical and pressure-sensitive properties of cement mortar with nanophase materials, Cement and Concrete research 34 (2004) 435-438.

[11] B. Han, Y. Wang, S. Dong, L. Zhang, S. Ding, X. Yu, J. Ou, Smart concretes and structures: A review, Journal of intelligent material systems and structures 26 (2015) 1303-1345. 
[12] T. Hou, J. Lynch, Conductivity-based strain monitoring and damage characterization of fiber reinforced cementitious structural components, in: Proc. SPIE, volume 5765, pp. 419-429.

[13] S. P. Shah, M. Konsta-Gdoutos, Z. Metaxa, P. Mondal, Nanoscale modification of cementitious materials, in: Nanotechnology in Construction 3, Springer, 2009, pp. 125-130.

[14] B. Han, X. Guan, J. Ou, Electrode design, measuring method and data acquisition system of carbon fiber cement paste piezoresistive sensors, Sensors and Actuators A: Physical 135 (2007) 360-369.

[15] B. Han, X. Yu, J. Ou, Multifunctional and smart carbon nanotube reinforced cement-based materials, in: Nanotechnology in civil infrastructure, Springer, 2011, pp. 1-47.

[16] B. Han, S. Ding, X. Yu, Intrinsic self-sensing concrete and structures: A review, Measurement 59 (2015) $110-128$.

[17] A. L. Materazzi, F. Ubertini, A. DAlessandro, Carbon nanotube cement-based transducers for dynamic sensing of strain, Cement and Concrete Composites 37 (2013) 2-11.

[18] F. Ubertini, S. Laflamme, H. Ceylan, A. L. Materazzi, G. Cerni, H. Saleem, A. D’ Alessandro, A. Corradini, Novel nanocomposite technologies for dynamic monitoring of structures: a comparison between cementbased embeddable and soft elastomeric surface sensors, Smart Materials and Structures 23 (2014) 045023.

[19] B. Zhang, Z. Zhou, K. Zhang, G. Yan, Z. Xu, Sensitive skin and the relative sensing system for real-time surface monitoring of crack in civil infrastructure, Journal of intelligent material systems and structures 17 (2006) 907-917.

[20] K. Loh, T. Hou, J. Lynch, N. Kotov, Nanotube-based sensing skins for crack detection and impact monitoring of structures, in: Proceedings of the 6th International Workshop on Structural Health Monitoring, Stanford, CA, USA.

[21] N. Hu, Y. Karube, M. Arai, T. Watanabe, C. Yan, Y. Li, Y. Liu, H. Fukunaga, Investigation on sensitivity of a polymer/carbon nanotube composite strain sensor, Carbon 48 (2010) 680-687.

[22] R. Howser, H. Dhonde, Y. Mo, Self-sensing of carbon nanofiber concrete columns subjected to reversed cyclic loading, Smart materials and structures (2011).

[23] M. S. Konsta-Gdoutos, C. A. Aza, Self sensing carbon nanotube (CNT) and nanofiber (CNF) cementitious composites for real time damage assessment in smart structures, Cement and Concrete Composites 53 (2014) $162-169$.

[24] A. D’Alessandro, M. Rallini, F. Ubertini, A. L. Materazzi, J. M. Kenny, Investigations on scalable fabrication procedures for self-sensing carbon nanotube cement-matrix composites for SHM applications, Cement and Concrete Composites 65 (2016) 200-213.

[25] B. Chen, K. Wu, W. Yao, Conductivity of carbon fiber reinforced cement-based composites, Cement and Concrete Composites 26 (2004) 291-297.

[26] M. Chiarello, R. Zinno, Electrical conductivity of self-monitoring CFRC, Cement and Concrete Composites 27 (2005) 463-469.

[27] S. Wen, D. Chung, Double percolation in the electrical conduction in carbon fiber reinforced cement-based materials, Carbon 45 (2007) 263-267.

[28] S. Wen, D. Chung, Carbon fiber-reinforced cement as a thermistor, Cement and Concrete Research 29 (1999) 961-965.

[29] H. Li, H. Xiao, J. Ou, Effect of compressive strain on electrical resistivity of carbon black-filled cementbased composites, Cement and Concrete Composites 28 (2006) 824-828.

[30] L. Chang, K. Friedrich, L. Ye, P. Toro, Evaluation and visualization of the percolating networks in multi-wall carbon nanotube/epoxy composites, Journal of materials science 44 (2009) 4003-4012.

[31] C. Feng, L. Jiang, Investigation of uniaxial stretching effects on the electrical conductivity of CNT-polymer nanocomposites, Journal of Physics D: Applied Physics 47 (2014) 405103. 
[32] N. Hu, H. Fukunaga, S. Atobe, Y. Liu, J. Li, Piezoresistive strain sensors made from carbon nanotubes based polymer nanocomposites, Sensors 11 (2011) 10691-10723.

[33] E. García-Macías, A. D’Alessandro, R. Castro-Triguero, D. Pérez-Mira, F. Ubertini, Micromechanics modeling of the electrical conductivity of carbon nanotube cement-matrix composites, Composites Part B: Engineering 108 (2017) 451-469.

[34] F. Deng, Q. Zheng, An analytical model of effective electrical conductivity of carbon nanotube composites, Applied Physics Letters 92 (2008) 071902.

[35] T. Takeda, Y. Shindo, Y. Kuronuma, F. Narita, Modeling and characterization of the electrical conductivity of carbon nanotube-based polymer composites, Polymer 52 (2011) 3852-3856.

[36] G. D. Seidel, D. C. Lagoudas, A micromechanics model for the electrical conductivity of nanotube-polymer nanocomposites, Journal of Composite Materials 43 (2009) 917-941.

[37] C. Feng, L. Jiang, Micromechanics modeling of the electrical conductivity of carbon nanotube (CNT)polymer nanocomposites, Composites Part A: Applied Science and Manufacturing 47 (2013) 143-149.

[38] C. Lin, H. Wang, W. Yang, Variable percolation threshold of composites with fiber fillers under compression, Journal of Applied Physics 108 (2010) 013509.

[39] T. Theodosiou, D. Saravanos, Numerical investigation of mechanisms affecting the piezoresistive properties of CNT-doped polymers using multi-scale models, Composites Science and Technology 70 (2010) 13121320 .

[40] T. Tallman, K. Wang, An arbitrary strains carbon nanotube composite piezoresistivity model for finite element integration, Applied Physics Letters 102 (2013) 011909.

[41] C. Feng, L. Jiang, Micromechanics modeling of bi-axial stretching effects on the electrical conductivity of CNT-polymer composites, International Journal of Applied Mechanics 7 (2015) 1550005.

[42] E. García-Macías, A. D’Alessandro, R. Castro-Triguero, D. Pérez-Mira, F. Ubertini, Micromechanics modeling of the uniaxial strain-sensing property of carbon nanotube cement-matrix composites for SHM applications, Composite Structures 163 (2017) 195-215.

[43] M. Park, H. Kim, J. P. Youngblood, Strain-dependent electrical resistance of multi-walled carbon nanotube/polymer composite films, Nanotechnology 19 (2008) 055705.

[44] S. Gong, Z. Zhu, S. Meguid, Carbon nanotube agglomeration effect on piezoresistivity of polymer nanocomposites, Polymer 55 (2014) 5488-5499.

[45] S. Wen, D. Chung, Damage monitoring of cement paste by electrical resistance measurement, Cement and Concrete Research 30 (2000) 1979-1982.

[46] J. Cao, D. Chung, Electric polarization and depolarization in cement-based materials, studied by apparent electrical resistance measurement, Cement and Concrete Research 34 (2004) 481-485.

[47] I. Kang, M. J. Schulz, J. H. Kim, V. Shanov, D. Shi, A carbon nanotube strain sensor for structural health monitoring, Smart materials and structures 15 (2006) 737-748.

[48] K. J. Loh, J. P. Lynch, B. Shim, N. Kotov, Tailoring piezoresistive sensitivity of multilayer carbon nanotube composite strain sensors, Journal of Intelligent Material Systems and Structures 19 (2008) 747-764.

[49] A. Materazzzi, F. Ubertini, A. DAlessandro, Carbon nanotube cement-based sensors for dynamic monitoring of concrete structures, in: Proceedings of the 2013 FIB Symposium, April, pp. 22-24.

[50] A. D’Alessandro, F. Ubertini, A. L. Materazzi, S. Laflamme, M. Porfiri, Electromechanical modelling of a new class of nanocomposite cement-based sensors for structural health monitoring, Structural Health Monitoring (2014) 1475921714560071.

[51] B. Han, K. Zhang, X. Yu, E. Kwon, J. Ou, Electrical characteristics and pressure-sensitive response measurements of carboxyl MWNT/cement composites, Cement and Concrete Composites 34 (2012) 794-800. 
[52] A. Sanli, C. Müller, O. Kanoun, C. Elibol, M. F.-X. Wagner, Piezoresistive characterization of multi-walled carbon nanotube-epoxy based flexible strain sensitive films by impedance spectroscopy, Composites Science and Technology 122 (2016) 18-26.

[53] S. Wansom, N. Kidner, L. Woo, T. Mason, AC-impedance response of multi-walled carbon nanotube/cement composites, Cement and Concrete Composites 28 (2006) 509-519.

[54] F. Verhulst, Nonlinear differential equations and dynamical systems, Springer Science \& Business Media, 2006.

[55] N. Swamy, G. Rigby, Dynamic properties of hardened paste, mortar and concrete, Matériaux et Construction 4 (1971) 13-40. 